\title{
Qualidade de vida de idosos com Insuficiência Renal Crônica (IRC): uma revisão integrativa da literatura
}

Quality of life of elderly people with Chronic Kidney Failure (CKI): an integrative literature review Calidad de vida de las personas mayores con Insuficiencia Renal Crónica (IRC): una revisión

Lorena Nayara Alves Neves ORCID: https://orcid.org/0000-0002-7271-4227

Centro Metropolitano da Amazônia, Brasil

E-mail: nayaralorena861@gmail.com

Camila Cordeiro de Santana Tavares ORCID: https://orcid.org/0000-0002-7354-6173

Universidade da Amazônia, Brasil

E-mail: camilacst93@gmail.com

Juliana Ferreira Rodrigues

ORCID: https://orcid.org/0000-0002-8114-9936

Universidade da Amazônia, Brasil

E-mail: julianafr998@gmail.com

Edilene Silva dos Santos

ORCID: https://orcid.org/0000-0002-4215-6180

Centro Metropolitano da Amazônia, Brasil

E-mail: edilenecsan@gmail.com

Francisco Hilângelo Vieira Barros

ORCID: https://orcid.org/0000-0002-2650-9724

Hospital Universitário Federal da Grande Dourados, Brasil

E-mail: fhvbqxda@yahoo.com.br

Antonia Gomes de Olinda

ORCID: https://orcid.org/0000-0002-5690-4506

Hospital Universitário Federal da Grande Dourados, Brasil

E-mail: antoniagomesdeolinda@gmail.com

Natasha Bezerra de Carvalho

ORCID: https://orcid.org/0000-0002-9101-7363

Hospital Universitário João de Barros Barretos, Brasil

E-mail: sasha_carvalho@yahoo.com.br

Eliane Moura da Silva

ORCID: https://orcid.org/0000-0002-2877-8987

Fundação Santa Casa de Misericórdia do Pará, Brasil

E-mail: eliane82moura@ hotmail.com

Maria Alcione Silva Gomes

ORCID: https://orcid.org/0000-0003-0662-2444 Hospital Universitário Federal da Grande Dourados, Brasil

E-mail: alcione_roseno@hotmail.com

Leonardo Oliveira Silva

ORCID: https://orcid.org/0000-0002-2172-8688 Hospital Universitário Federal da Grande Dourados, Brasil

E-mail: leonardo-oliveira-s@ hotmail.com

Jaqueline Bernal

ORCID: https://orcid.org/0000-0002-5909-8319 Hospital Universitário Federal da Grande Dourados, Brasil

E-mail: jaquebernal@hotmail.com

Ana Gabriela Martins Dias

ORCID: https://orcid.org/0000-0002-5767-1097

Universidade da Amazônia Brasil

E-mail: gabriela.mdias15@gmail.com

Thanaira Aicha Fernandes Maciel

ORCID: https://orcid.org/0000-0002-4119-6148

Universidade do Estado do Pará, Brasil

E-mail: aichafernandes1@gmail.com

Robson Luis Souza Monteiro

ORCID: https://orcid.org/0000-0001-5283-0004 


\author{
Universidade da Amazônia, Brasil \\ E-mail: Robson.prof@gmail.com \\ Gyselle Morais da Silva \\ ORCID: https://orcid.org/0000-0001-8632-5364 \\ Escola Superior da Amazôia, Brasil \\ Email: gysagt2@hotmail.com \\ Gabrielle Cecília Lobo da Costa \\ ORCID: https://orcid.org/0000-0001-5234-6343 \\ Escola Superior da Amazônia, Brasil \\ E-mail: gabriellelobovaladares0405@gmail.com \\ Cintia Maria da Silva Gomes \\ ORCID: https://orcid.org/0000-0002-4620-3765 \\ Universidade do Estado do Pará, Brasil \\ E-mail: gcintia1980@gmail.com \\ Viviane Ferraz Ferreira de Aguiar \\ ORCID: https://orcid.org/0000-0003-3025-1065 \\ Universidade Federal do Pará, Brasil \\ E-mail: viviane.ferraz29@gmail.com \\ Mônica Custódia do Couto Abreu Pamplona \\ ORCID: https://orcid.org/0000-0002-8508-1019 \\ Universidade do Estado do Pará, Brasil \\ E-mail: custodiaabreu@ hotmail.com \\ Dayara de Nazaré Rosa de Carvalho \\ ORCID: https://orcid.org/0000-0001-8569-3392 \\ Universidade do Estado do Pará, Brasil \\ E-mail: dayara_twain@hotmail.com \\ Ivonete Vieira Pereira Peixoto \\ ORCID: https://orcid.org/0000-0002-5463-9630 \\ Universidade do Estado do Pará, Brasil \\ E-mail: ivonetep@hotmail.com
}

\begin{abstract}
Resumo
Objetivo: Analisar na literatura nacional e internacional a qualidade de vida de idosos com Insuficiência Renal Crônica no período de 2015 a 2020. Método: Este estudo se trata de uma pesquisa descritiva, qualitativa, do tipo Revisão Integrativa da Literatura (RIL). Para a exploração do material e categorização dos estudos utilizou-se o software IRaMuTeQ. Resultados: obteve-se a formação de 4 classes, sendo elas: A dimensão e fatores que interferem na qualidade de vida de idosos renais crônicos; As limitações e restrições ocasionadas pelo processo hemodialítico; Qualidade de vida de pacientes idosos com IRC associado a diálise e A comunicação entre familiares e profissionais para prestação do cuidado. Incorporou-se o conteúdo através da Classificação Hierárquica Descendente, por meio de um dendrograma, elaborado pelos autores, em que representa a frequências das palavras (f) e o valor do qui-quadrado (x2). Conclusão: A qualidade de vida de idosos com IRC é um aspecto importante, pois estes pacientes possuem limitações relacionadas a doença e também ao tratamento por hemodiálise. Por isso, este estudo aborda subsídios para que profissionais de saúde possam identificar os fatores que interferem na qualidade de vida de idosos com IRC para assim se ter uma evolução positiva e um tratamento adequado.
\end{abstract}

Palavras-chave: Cuidados de enfermagem; Idoso; Insuficiência renal crônica; Diálise renal; Qualidade de vida.

\begin{abstract}
Objective: To analyze in the national and international literature the quality of life of elderly people with Chronic Renal Failure in the period from 2015 to 2020. Method: This study is a descriptive, qualitative, type of Integrative Literature Review (RIL). To explore the material and categorize the studies, the IRaMuTeQ software was used. Results: 4 classes were formed, namely: The size and factors that affect the quality of life of elderly with chronic kidney disease; The limitations and restrictions caused by the hemodialysis process; Quality of life of elderly patients with CRF associated with dialysis and Communication between family members and professionals to provide care. The content was incorporated through the Descending Hierarchical Classification, by means of a dendrogram, prepared by the authors, in which it represents the frequency of the words (f) and the chi-square value (x2). Conclusion: The quality of life of elderly people with CRF is an important aspect, as these patients have limitations related to the disease and also to treatment by hemodialysis. For this reason, this study addresses subsidies so that health professionals can identify the factors that interfere in the quality of life of elderly people with CRF in order to have a positive evolution and an appropriate treatment.
\end{abstract}

Keywords: Nursing care; Old man; Chronic renal failure; Renal dialysis; Quality of life.

\title{
Resumen
}

Objetivo: Analizar en la literatura nacional e internacional la calidad de vida de los ancianos con Insuficiencia Renal Crónica en el período de 2015 a 2020. Método: Este estudio es un tipo de Revisión de Literatura Integrativa (EIR) descriptiva, cualitativa. Para explorar el material y categorizar los estudios, se utilizó el software IRaMuTeQ. 
Resultados: Se conformaron 4 clases, a saber: El tamaño y factores que afectan la calidad de vida de los ancianos con enfermedad renal crónica; Las limitaciones y restricciones provocadas por el proceso de hemodiálisis; Calidad de vida de los pacientes ancianos con IRC asociada a diálisis y Comunicación entre familiares y profesionales para la atención. El contenido se incorporó mediante la Clasificación Jerárquica Descendente, mediante un dendrograma, elaborado por los autores, en el que representa la frecuencia de las palabras (f) y el valor de chi-cuadrado (x2). Conclusión: La calidad de vida de los ancianos con IRC es un aspecto importante, ya que estos pacientes tienen limitaciones relacionadas con la enfermedad y también con el tratamiento por hemodiálisis. Por ello, este estudio aborda las subvenciones para que los profesionales sanitarios puedan identificar los factores que interfieren en la calidad de vida de las personas mayores con IRC para tener una evolución positiva y un tratamiento adecuado.

Palabras clave: Atención de enfermería; Anciano; Falla renal cronica; Diálisis renal; Calidad de vida.

\section{Introdução}

O envelhecimento populacional é um fenômeno mundial decorrente da elevação da expectativa de vida e diminuição da taxa de natalidade, devido a melhorias nas condições de vida e saúde dos indivíduos. Em 2019, no Brasil, o número de idosos ultrapassou os 29 milhões e a expectativa é que, até 2060, este número suba para 73 milhões de idosos com 60 anos ou mais, representando um aumento de 160\% (Brasil, 2019).

O envelhecimento acarreta modificações físicas, mentais e socioeconômicas, tornando os idosos mais vulneráveis às diversas patologias, interferindo assim em sua qualidade de vida (Menezes et al., 2018). Dentre as doenças que mais acometem os idosos, encontram-se as Doenças Crônicas Não Transmissíveis (DCNT), sendo a Hipertensão Arterial Sistêmica (HAS), Diabetes Mellitus (DM) e as Insuficiências Renais Crônicas (IRC) as de maior incidência nessa população (Barreto et al.,2015).

Insuficiência Renal Crônica (IRC) é uma afecção gradual que acometem os rins, que tem a função de equilibrar e filtrar os metabólitos gerados no sangue, na qual é definida como uma perda ou disfunção gradual e progressiva da função renal, em que se terá a diminuição da Taxa de Filtração Glomerular (TFG), provocando assim o acúmulo de toxinas no sangue (Oliveira et al., 2019).

No Brasil, estimativas apontam que no ano de 2006 existiam cerca de 1,75 milhões de usuários do sistema de saúde que eram portadores de IRC, e posteriormente no ano de 2009, essa estimativa ultrapassou 400 portadores por milhões de habitantes na população. A Doença Renal possui um estágio final que é designado como IRC na qual, nesse estágio o paciente necessita de uma terapia renal que vai proporcionar a sobrevida do usuário. Umas das terapias que podem ser aplicadas aos pacientes Idosos com IRC é designada de hemodiálise e é predominante no Brasil com o elevado número de pacientes no país (Santos et al., 2018).

Segundo o estudo de Souza et al (2017) a terapêutica hemodialítica e o progresso da doença renal afeta diretamente a qualidade de vida do idoso pois, causa limitações, prejuízos na saúde física, mental e funcional. Desta forma, é importante compreender que o termo qualidade de vida está correlacionado ao estado de saúde e a capacidade do indivíduo de viver plenamente.

Diante do exposto, foi desenvolvida uma Revisão Integrativa da Literatura (RIL) com o objetivo de analisar na literatura nacional e internacional a qualidade de vida de idosos com Insuficiência Renal Crônica no período de 2015 a 2020, visto que esse estudo irá contribuir para a melhoria no atendimento a indivíduos que estejam nessa situação.

\section{Metodologia}

Trata-se de uma pesquisa descritiva, qualitativa, do tipo Revisão Integrativa da Literatura (RIL). Esse tipo de revisão é realizado por meio de diferentes etapas apresentadas a seguir: 1) Identificação do tema e seleção da questão de pesquisa; 2) Estabelecimento de critérios de inclusão e exclusão de estudos; 3) Categorização dos estudos; 4) Avaliação dos estudos; 5) Interpretação dos resultados; 6) Apresentação da Revisão Integrativa da Literatura (Seabra et al., 2019). 
A partir do objeto de estudo, delimitou-se a seguinte questão norteadora, por meio da estratégia de PICo (P: Paciente, I: Intervenção, Co: Contexto) "Como é realizada a avaliação da qualidade de vida de idosos com Insuficiência Renal Crônica (IRC)? Quais fatores que interferem na qualidade de vida desses idosos?

A coleta de dado foi realizada no mês de dezembro de 2020, através de acesso online nas bases e bancos de dados a seguir: Biblioteca Virtual de Saúde (BVS), PubMed/ Medline (National Library of Medicine and National Institutes of Health/ Medical Literature Analysi Sand Retrieval System Online) e ScientificElectronic Library Online (SCIELO). Para otimizar e refinar a busca e garantir o direcionamento para todos os trabalhos relevantes, a seleção dos artigos ocorreu a partir da combinação de três descritores cadastrados no DeCS/MeSH (Descritores em Ciências da Saúde/Medical SubjectHeading) respectivamente: "Cuidados de Enfermagem", "Idoso", "Insuficiência renal crônica", "Diálise renal" e "Qualidade de vida" mediados pelo operador booleano "AND" e "OR"

Na chave de combinação de pesquisa foi composta pela seguinte combinação na BVS: (tw:(cuidados de enfermagem)) AND (tw:( idoso)) AND (tw:( insuficiência renal crônica)) AND (tw:(diálise renal)) AND (tw:(qualidade de vida)) AND (fulltext:("1") AND db:("MEDLINE" OR "BDENF" OR "IBECS" OR "LILACS") AND la:("en" OR "pt" OR "es")) AND (year_cluster: [2015 TO 2020]).

Os critérios de inclusão utilizados para a elaboração da RIL foram: artigos completos publicados no período de 2015 a 2020, com o objetivo de excluir estudos defasados, estudos disponíveis em periódicos com acesso gratuito nos idiomas português, inglês e espanhol, e que apresentassem significância relativa ao tema de estudo. Foram excluídos artigos fora do período delimitado, cartas ao editor, estudos reflexivos, editoriais, artigos incompletos, artigos pagos e, os que não estivesse de acordo com a temática deste estudo.

Na coleta de dados, a partir dos critérios de inclusão e exclusão estabelecidos, foi utilizado o instrumento adaptado de URSI com o objetivo de extrair os dados relevantes, diminuir erros na transcrição e garantir as informações contidas nos artigos selecionados, título do artigo, autor/ano, objetivos e a síntese dos principais resultados (Ursi, 2005).

Para a análise de conteúdo, utilizou-se o conteúdo proposto por Lawrence Bardin que se subdividi em três etapas sendo: pré-análise, exploração do material e a abordagem dos resultados obtidos e interpretados tendo a finalidade de propor uma apreciação critica das análises de conteúdo nas pesquisas qualitativas e quantitativas (Bardin, 2016).

Em seguida, para a exploração do material e categorização dos estudos utilizou-se o software IRaMuTeQ (Interface de $R$ pour lês Analyses Multidimensionnelles de Textes et de 101 Questionnaires), o qual foi desenvolvido por Pierre Ratinaud na França. O programa se ancora no software R e na linguagem Python que possibilita diferentes formas de análise sobre o corpus textuais que é criado para o início da análise (Camargo et al., 2013).

A criação do corpus textuais, para a exploração e categorização do estudo, se deu a partir dos dados existentes nos resultados e conclusões dos artigos selecionados no estudo, haja vista que o conteúdo criado deve ser colocado em um texto único sem formação para que o software faça a leitura adequada conforme o Quadro 1.

Quadro 1: Codificação dos corpus textuais para análise no IRaMuTeQ.

\begin{tabular}{|c|l|}
\hline \multicolumn{1}{|c|}{ Codificação do artigo } & \multicolumn{1}{|c|}{ Codificação da base e banco de dados } \\
\hline$*$ Art_1 a *Art_n ${ }^{\circ}$ (artigos selecionados) & Base_1:BVS \\
& Base_2:PubMed \\
& Base_3:SCIELO \\
\hline
\end{tabular}

Fonte: Autores (2021). 


\section{Resultados e Discussão}

Obteve-se, ao final da coleta de dados, um total de 318 estudos nas referidas bases e banco de dados. Após essa fase os estudos passaram por avaliação quanto ao título e resumo e deste total, 180 foram excluídos do processo de triagem. A apreciação durante essa fase teve o propósito de descartar estudos que não atenderam aos critérios de elegibilidade. Ao final do processo de triagem emergiu a amostragem final, a partir da leitura do texto completo, composta de 16 estudos que foram avaliados quanto ao título do artigo, autores, ano de publicação e base de dados, objetivo e síntese das considerações finais e aqueles que respondiam à questão de pesquisa. O processo de seleção dos estudos selecionados está exposto no fluxograma de busca a seguir (Figura 1).

Figura 1: Fluxograma de busca e seleção de documentos.
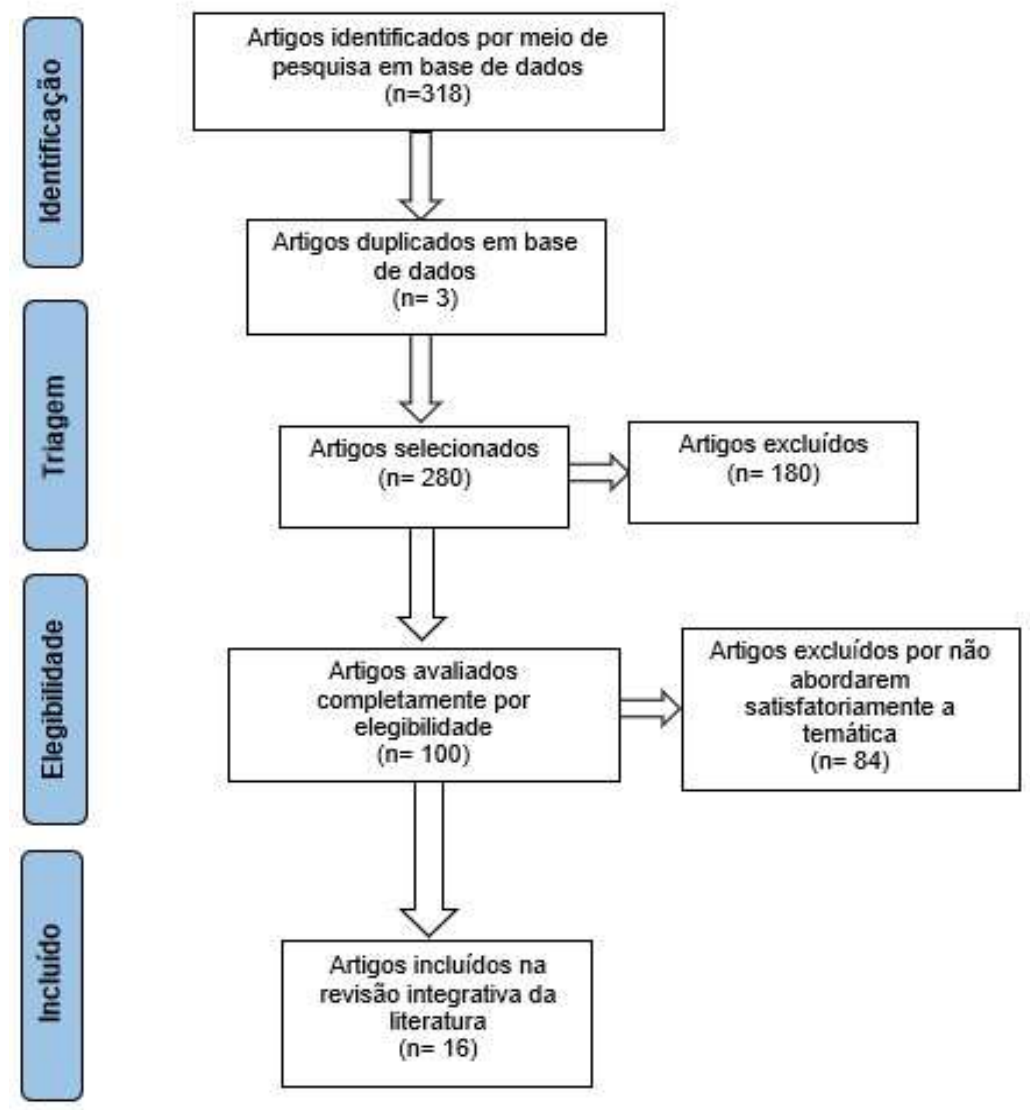

Fonte: autores da pesquisa (2021).

Na síntese dos artigos selecionados na RIL, de acordo com o Quadro 2, os principais assuntos abordados foram relacionados aos cuidados prestados aos pacientes que possuem a insuficiência renal crônica, a promoção da qualidade de vida e os instrumentos necessários e os malefícios que os pacientes com insuficiência renal crônica desenvolvem no tratamento dialítico. 
Quadro 2: Identificação e síntese dos artigos selecionados.

\begin{tabular}{|c|c|c|c|c|}
\hline $\begin{array}{c}\text { Base de } \\
\text { dados }\end{array}$ & Título & Autoria/ Ano & Objetivos & Resumo das considerações finais \\
\hline SCIELO & $\begin{array}{l}\text { Coping e qualidade de vida } \\
\text { de pacientes em } \\
\text { hemodiálise. }\end{array}$ & $\begin{array}{l}\text { Lira CLOB, } \\
\text { Avelar TC, } \\
\text { Bueno } \\
\text { JMMH. } 2015 .\end{array}$ & $\begin{array}{l}\text { Investigar a relação entre estratégias } \\
\text { de coping e qualidade de vida de } \\
\text { pacientes em hemodiálise. }\end{array}$ & $\begin{array}{l}\text { O estudo contribuiu com dados } \\
\text { importantes para a reflexão e } \\
\text { planejamento da prática } \\
\text { profissional com pacientes com } \\
\text { insuficiência renal crônica e os em } \\
\text { tratamento hemodialítico em } \\
\text { particular. }\end{array}$ \\
\hline SCIELO & $\begin{array}{l}\text { Ações da assistência de } \\
\text { enfermagem ao portador de } \\
\text { insuficiência renal crônica } \\
\text { em tratamento } \\
\text { hemodialítico. }\end{array}$ & $\begin{array}{l}\text { Negreiros } \\
\text { PIR, Siqueira } \\
\text { TDA. } 2016 .\end{array}$ & $\begin{array}{l}\text { Descrever as ações da assistência em } \\
\text { enfermagem ao portador de } \\
\text { insuficiência renal crônica em } \\
\text { tratamento hemodialítico. }\end{array}$ & $\begin{array}{l}\text { A assistência de enfermagem ao } \\
\text { paciente renal crônico em } \\
\text { tratamento hemodialítico não se } \\
\text { resume apenas nos cuidados } \\
\text { técnicos, é importante que o } \\
\text { enfermeiro preste um cuidado } \\
\text { integral, percebendo o paciente } \\
\text { como um ser único. }\end{array}$ \\
\hline PubMed & $\begin{array}{l}\text { Association of Nondisease- } \\
\text { Specific Problems with } \\
\text { Mortality, Long-Term Care, } \\
\text { and Functional Impairment } \\
\text { among Older Adults Who } \\
\text { Require Skilled Nursing } \\
\text { Care after Dialysis } \\
\text { Initiation }\end{array}$ & $\begin{array}{l}\text { Bowling CB, } \\
\text { Plantinga L, } \\
\text { Hall RK et al., } \\
2016 .\end{array}$ & $\begin{array}{l}\text { Examinar a associação da carga de } \\
\text { problemas não específicos de } \\
\text { coocorrência com mortalidade, } \\
\text { transição para cuidados de longo } \\
\text { prazo (LTC) e comprometimento } \\
\text { funcional entre idosos que } \\
\text { necessitaram de cuidados pós-agudos } \\
\text { de SNF após o início da diálise. }\end{array}$ & $\begin{array}{l}\text { A presença de múltiplos problemas } \\
\text { entre idosos novos para diálise } \\
\text { esteve associada a maior risco de } \\
\text { mortalidade, transição do SNF para } \\
\text { LTC e comprometimento funcional. }\end{array}$ \\
\hline SCIELO & $\begin{array}{l}\text { A dinâmica familiar frente } \\
\text { ao idoso em tratamento pré- } \\
\text { dialítico. }\end{array}$ & $\begin{array}{l}\text { Jacobi CS, } \\
\text { Beuter M, } \\
\text { Perlini } \\
\text { NMOG et al., } \\
2017 .\end{array}$ & $\begin{array}{l}\text { Descrever a dinâmica da família que } \\
\text { convive com um idoso em tratamento } \\
\text { pré-dialítico. }\end{array}$ & $\begin{array}{l}\text { Este estudo poderá contribuir para } \\
\text { promover discussões que abordem } \\
\text { o cuidado prestado pela equipe de } \\
\text { saúde, que atende a idosos com } \\
\text { IRC em ambulatórios e na atenção } \\
\text { básica, visando a um atendimento } \\
\text { qualificado. }\end{array}$ \\
\hline BVS & $\begin{array}{l}\text { Análise da qualidade de } \\
\text { vida do paciente na fase de } \\
\text { pré-dialise. }\end{array}$ & $\begin{array}{l}\text { Parrado MCR, } \\
\text { Pozo MG, } \\
\text { Garrido MC } \\
\text { et al., } 2017 .\end{array}$ & $\begin{array}{l}\text { Analisar a Qualidade de Vida } \\
\text { Relacionada à Saúde de pacientes } \\
\text { com Doença Renal Crônica } \\
\text { Avançada no estágio } 5 .\end{array}$ & $\begin{array}{l}\text { Os pacientes com Doença Renal } \\
\text { Crônica Avançada estágio } 5 \\
\text { diminuíram sua Qualidade de Vida } \\
\text { Relacionada situação de emprego, } \\
\text { função sexual, função física e saúde } \\
\text { geral. }\end{array}$ \\
\hline SCIELO & $\begin{array}{l}\text { Qualidade de vida de idosos } \\
\text { com doença renal crônica } \\
\text { em tratamento conservador. }\end{array}$ & $\begin{array}{l}\text { Pereira RMP, } \\
\text { Batista MA, } \\
\text { Meira AS et }\end{array}$ & $\begin{array}{l}\text { Descrever a qualidade de vida (QV) } \\
\text { de idosos com Doença Renal Crônica } \\
\text { (DRC) em tratamento conservador, }\end{array}$ & $\begin{array}{l}\text { Foi demostrado que uma negativa } \\
\text { qualidade de vida está relacionada } \\
\text { com um maior número de }\end{array}$ \\
\hline
\end{tabular}




\begin{tabular}{|c|c|c|c|c|}
\hline & & al., 2017. & $\begin{array}{l}\text { correlacionando-a com aspectos } \\
\text { sociodemográficos e de saúde. }\end{array}$ & $\begin{array}{l}\text { complicações correlacionado com o } \\
\text { número de comorbidades. É preciso } \\
\text { levar em conta a terapêutica as } \\
\text { complicações da DRC, pois } \\
\text { diminuindo a sintomatologia se } \\
\text { beneficiará a qualidade de vida. }\end{array}$ \\
\hline BVS & $\begin{array}{l}\text { Associação entre } \\
\text { características } \\
\text { sociodemográficas e } \\
\text { qualidade de vida de } \\
\text { pacientes renais crônicos } \\
\text { em hemodiálise. }\end{array}$ & $\begin{array}{l}\text { Marinho } \\
\text { CLA, Oliveira } \\
\text { JV, Borges } \\
\text { JES et } \\
\text { al.,2017. }\end{array}$ & $\begin{array}{l}\text { Associar os domínios de qualidade de } \\
\text { vida com as características } \\
\text { sociodemográficas de pacientes } \\
\text { renais crônicos. }\end{array}$ & $\begin{array}{l}\text { A utilização de instrumentos } \\
\text { capazes de mensurar a qualidade de } \\
\text { vida é bastante útil, sendo o } \\
\text { KDQOL-SFTM } 1.3 \text { uma ferramenta } \\
\text { de fácil acesso e baixo custo que } \\
\text { permite o conhecimento pela } \\
\text { equipe de enfermagem dos déficits } \\
\text { de qualidade de vida, facilitando } \\
\text { assim, sua intervenção em busca de } \\
\text { melhorias para estes pacientes. }\end{array}$ \\
\hline BVS & $\begin{array}{l}\text { Qualidade de vida de } \\
\text { pacientes com insuficiência } \\
\text { renal em tratamento } \\
\text { hemodialítico. }\end{array}$ & $\begin{array}{l}\text { Silva KAL, } \\
\text { Cargnin MCS, } \\
\text { Ventura J et } \\
\text { al., } 2017 .\end{array}$ & $\begin{array}{l}\text { Avaliar a qualidade de vida de } \\
\text { pessoas com Insuficiência Renal } \\
\text { Crônica em tratamento hemodialítico } \\
\text { por meio do instrumento KDQOL- } \\
\text { SFTM1.3. }\end{array}$ & $\begin{array}{l}\text { O instrumento pode auxiliar a } \\
\text { equipe de saúde no planejamento } \\
\text { do cuidado e na implementação de } \\
\text { ações específicas e na melhora da } \\
\text { qualidade de vida. }\end{array}$ \\
\hline BVS & $\begin{array}{l}\text { Pacientes em hemodiálise: } \\
\text { importância do autocuidado } \\
\text { com a fístula arteriovenosa. }\end{array}$ & $\begin{array}{l}\text { Clementino } \\
\text { DC, Souza } \\
\text { AMQ, Barros } \\
\text { DCC et al., } \\
2018 .\end{array}$ & $\begin{array}{l}\text { Averiguar o conhecimento dos } \\
\text { pacientes com doença renal crônica } \\
\text { acerca do autocuidado com a fístula } \\
\text { artéria venosa (FAV). }\end{array}$ & $\begin{array}{l}\text { Estimular a prática do autocuidado } \\
\text { pela equipe de enfermagem junto } \\
\text { aos doentes renais crônicos acerca } \\
\text { de sua doença, manifestações } \\
\text { clínicas, estilo de vida, tratamento e } \\
\text { cuidados com a FAV, uma vez que } \\
\text { esta prática melhorar a qualidade de } \\
\text { vida dos pacientes. }\end{array}$ \\
\hline BVS & $\begin{array}{l}\text { Prurido no paciente em } \\
\text { hemodiálise: associação } \\
\text { com ingestão de fósforo e } \\
\text { nível sérico de cálcio. }\end{array}$ & $\begin{array}{l}\text { Tinôco JDS, } \\
\text { Paiva } \\
\text { MGMN, } \\
\text { Macedo BM } \\
\text { et al., } 2018 .\end{array}$ & $\begin{array}{l}\text { Analisar a associação entre a } \\
\text { ocorrência do prurido e a adesão à } \\
\text { dieta prescrita, indicadores } \\
\text { bioquímicos da função renal e a } \\
\text { qualidade da hemodiálise, em } \\
\text { pacientes renais crônicos. }\end{array}$ & $\begin{array}{l}\text { O prurido em pacientes renais } \\
\text { crônicos submetidos à hemodiálise } \\
\text { sofre influência da não adesão } \\
\text { adequada à dieta prescrita, além da } \\
\text { elevação de indicadores } \\
\text { bioquímicos da função renal. }\end{array}$ \\
\hline BVS & $\begin{array}{l}\text { Relação entre qualidade de } \\
\text { vida relacionada à saúde e } \\
\text { ansiedade/depressão em } \\
\text { pacientes com hemodiálise } \\
\text { crônica. }\end{array}$ & $\begin{array}{l}\text { Martínez } \\
\text { RMM, } \\
\text { Gallego NM, } \\
\text { Cabezas AMS } \\
\text { et al., } 2019 .\end{array}$ & $\begin{array}{l}\text { Analisar a qualidade de vida } \\
\text { relacionada à saúde em pacientes } \\
\text { com hemodiálise e sua relação com } \\
\text { ansiedade e depressão. }\end{array}$ & $\begin{array}{l}\text { Os pacientes em hemodiálise têm } \\
\text { pior qualidade de vida } \\
\text { especialmente nas dimensões Carga } \\
\text { da Doença Renal, Sono, Saúde } \\
\text { Geral, Vitalidade, Função Física e } \\
\text { Papel Físico tendo a probabilidade } \\
\text { de desenvolver ansiedade e/ou } \\
\text { depressão. }\end{array}$ \\
\hline
\end{tabular}




\begin{tabular}{|c|c|c|c|c|}
\hline PubMed & $\begin{array}{l}\text { Multidimensional pruritus } \\
\text { assessment in hemodialysis } \\
\text { patients }\end{array}$ & $\begin{array}{l}\text { Ersoy NA, } \\
\text { Akyar I. } 2019 .\end{array}$ & $\begin{array}{l}\text { Determinar a prevalência de prurido } \\
\text { urêmico e investigar o impacto } \\
\text { multidimensional em pacientes com } \\
\text { hemodiálise. }\end{array}$ & $\begin{array}{l}\text { A avaliação multidimensional faz- } \\
\text { se necessária pois apoiam a } \\
\text { necessidade de desenvolvimento do } \\
\text { manejo padronizado e específico } \\
\text { dos sintomas do paciente. }\end{array}$ \\
\hline PubMed & $\begin{array}{l}\text { Quality of life, symptoms, } \\
\text { and sleep quality of elderly } \\
\text { with end-stage renal disease } \\
\text { receiving conservative } \\
\text { management: a systematic } \\
\text { review }\end{array}$ & $\begin{array}{l}\text { Ren Q, Shi Q, } \\
\text { Ma T et al., } \\
2019 .\end{array}$ & $\begin{array}{l}\text { Examinar a qualidade de vida, os } \\
\text { sintomas e a qualidade do sono dos } \\
\text { pacientes idosos com ESRD em } \\
\text { gestão conservadora. }\end{array}$ & $\begin{array}{l}\text { Apesar dos estudos em literaturas } \\
\text { serem limitados, o estudo mostrou } \\
\text { que a gestão conservadora } \\
\text { melhorará a qualidade de vida e } \\
\text { aliviará os sintomas de pacientes } \\
\text { com doença renal em estágio } \\
\text { terminal com implicações clínicas } \\
\text { consideráveis principalmente em } \\
\text { pacientes idosos. }\end{array}$ \\
\hline PubMed & $\begin{array}{l}\text { Family Perceptions of } \\
\text { Quality of End-of-Life Care } \\
\text { for Veterans with Advanced } \\
\text { CKD }\end{array}$ & $\begin{array}{l}\text { Richards CA, } \\
\text { Liu CF, } \\
\text { Hebert PL et } \\
\text { al., } 2019 .\end{array}$ & $\begin{array}{l}\text { Descrever a relação entre padrões de } \\
\text { cuidados de fim de vida e tratamento } \\
\text { de diálise com qualidade de } \\
\text { assistência ao fim de vida relatada } \\
\text { pela família nesta população }\end{array}$ & $\begin{array}{l}\text { O cuidado focado na extensão da } \\
\text { vida em vez de conforto foi } \\
\text { associado a menores classificações } \\
\text { familiares de cuidados de fim de } \\
\text { vida, independentemente de os } \\
\text { pacientes terem recebido diálise. }\end{array}$ \\
\hline BVS & $\begin{array}{l}\text { Percepção de pacientes } \\
\text { renais crônicos em } \\
\text { hemodiálise sobre } \\
\text { transplante renal. }\end{array}$ & $\begin{array}{l}\text { Silva GL, } \\
\text { Lemos KCR, } \\
\text { Barbosa AO } \\
\text { et al., } 2020 .\end{array}$ & $\begin{array}{l}\text { Identificar a percepção de pacientes } \\
\text { com doença renal crônica em } \\
\text { hemodiálise sobre transplante renal. }\end{array}$ & $\begin{array}{l}\text { Constatou-se que a percepção dos } \\
\text { entrevistados sobre o transplante } \\
\text { renal se relacionou à mudança de } \\
\text { vida, garantindo sensação de } \\
\text { liberdade em relação a outras } \\
\text { terapias e aumento da expectativa } \\
\text { de vida. }\end{array}$ \\
\hline PubMed & $\begin{array}{l}\text { Quality of Life and Social } \\
\text { Support of People on } \\
\text { Peritoneal Dialysis: Mixed } \\
\text { Methods Research }\end{array}$ & $\begin{array}{l}\text { Suñer MS, } \\
\text { Soler RS, } \\
\text { Plana AM et } \\
\text { al., } 2020 .\end{array}$ & $\begin{array}{l}\text { Estudar a qualidade de vida } \\
\text { relacionada à saúde, o apoio social } \\
\text { percebido e as experiências das } \\
\text { pessoas que recebem diálise } \\
\text { peritoneal. }\end{array}$ & $\begin{array}{l}\text { Os menores escores de qualidade } \\
\text { de vida relacionados à saúde } \\
\text { corresponderam à percepção de } \\
\text { saúde geral, vitalidade e papéis } \\
\text { físicos. O apoio social percebido } \\
\text { pelos participantes foi ideal, e esse } \\
\text { apoio tem sido associado, física e } \\
\text { mentalmente, a uma melhor } \\
\text { percepção de qualidade de vida. }\end{array}$ \\
\hline
\end{tabular}

Fonte: Autores (2021).

A partir da análise utilizando o software IRaMuTeQ (Interface de R pour les Analyses Multidimensionnelles de Textes et de Questionnaires) o resultado incorporou-se o conteúdo através da CHD (Classificação Hierárquica Descendente), por meio de um dendrograma, elaborado pelos autores, em que representa a frequências das palavras (f) e o valor do quiquadrado (x2). Teve-se a formação das classes, dividas e identificadas, por cores e porcentagens. A classe 1 (Vermelha) corresponde a $26,6 \%$ das palavras no corpus, a classe 2 (Verde) corresponde a 12,5\% das palavras, a classe 3 (azul) 
corresponde a $25,0 \%$ da associação de palavras no corpus e a classe 4 (Roxo) corresponde a 35,9\% da associação de palavras no corpus.

Após a análise da identificação dos domínios textuais e interpretação buscou-se nomear seus respectivos significados nas classes descritas abaixo: 1) A dimensão e fatores que interferem na qualidade de vida de idosos renais crônicos; 2) As limitações e restrições ocasionadas pelo processo hemodialítico; 3) Qualidade de vida de pacientes idosos com IRC associado a dialise; 4) A comunicação entre familiares e profissionais para prestação do cuidado. O dendrograma de classes está exposto a seguir (Figura 2).

Figura 2: Dendrograma realizado a partir da análise de CHD está exposta na Figura a seguir.

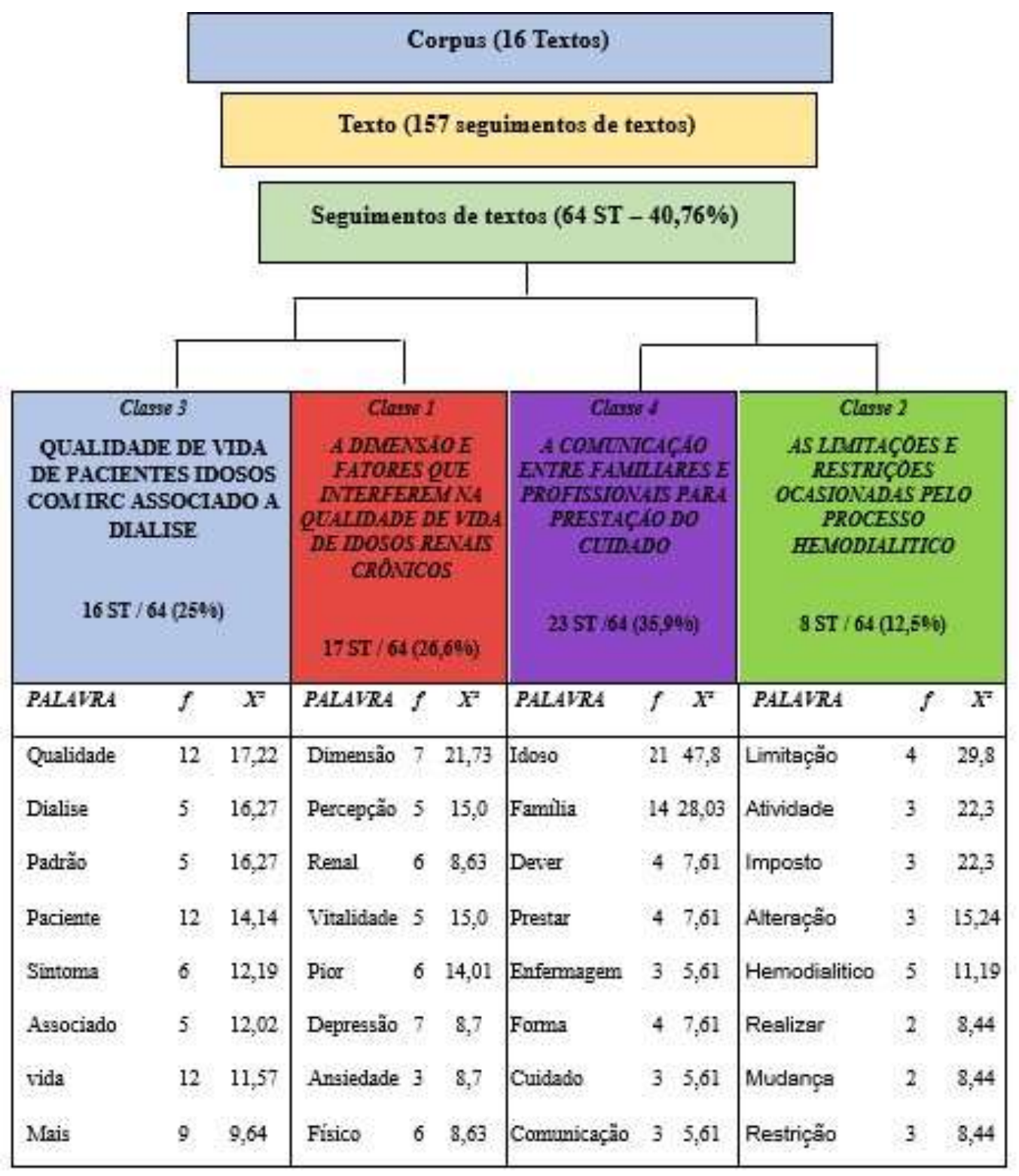

Fonte: Autores (2021).

\section{Classe 1: A dimensão e fatores que interferem na qualidade de vida de idosos renais crônicos}

Conforme a classe $1(26,6 \%)$, que através da análise emergiu, a categoria: A dimensão e fatores os que interferem na qualidade de vida de idosos renais crônicos, observou-se que as palavras de maior frequência mostrada no dendrograma são: dimensão, percepção, renal, vitalidade, pior, depressão, ansiedade e físico. 
A Terapia Renal Substitutiva (TRS) do tipo hemodiálise repercutem negativamente na qualidade de vida dos idosos com IRC haja vista que esses fatores estão relacionados a aspectos físicos, emocionais e psicológico, podendo exemplificar tais como: restrições hídricas, afastamento do trabalho, limitações físicas, declínio sexual entre outras (Jesus et al., 2019).

Ademais, em dimensão psicológica, a população idosa submetida a diálise enfrenta reações adversas vindas do tratamento que podem propiciar o desenvolvimento de sintomas depressivos reduzindo assim, a adesão ao tratamento e a piora da evolução da patologia. Desta maneira, segundo o estudo de Júnior et al (2018) é notado que a sintomatologia dos pacientes com IRC acometidos por transtornos psiquiátricos estão relacionados a anorexia pois é uma condição incomum nos pacientes em tratamento conservador afetando assim a qualidade de vida e a maior probabilidade da ocorrência do suicido.

No estudo de Pretto et al (2020) foi constatado que a depressão não tem indicativo associado a complicações a pacientes com IRC, porém tem relação com as intercorrências e sintomas após o processo hemodialítico pois, os sintomas desenvolvidos no paciente são em decorrência da patologia e tratamento, levando assim a severidade dos distúrbios emocionais, considerando-se que os sintomas repercutem na intensificação da carga psicológica e emocional, tendo associação aos sintomas depressivos.

A prática da atividade para pacientes com IRC gera efeitos positivos e significativos no desenvolvimento da capacidade funcional do idosos, função muscular e consequentemente a melhora na qualidade de vida. Entretanto, apesar das vantagens oferecidas por meio da prática periódica de atividade físicas, há estudos que mostram que pacientes em tratamento hemodialítico apresentam baixos níveis na pratica de atividade física, contribuindo assim para o surgimento do sedentarismo e deficiências a nível funcional (Fukushima et al., 2018).

Sabe-se que o processo de envelhecimento senescente acarretas mudanças no organismo dos idosos, podendo exemplificar que uma dessas alterações, estão relacionadas a perda fisiológica da massa muscular, há vista que essas alterações podem serem acentuadas através da inatividade física. Assim, no estudo de Hirai et al (2016) observa-se que pacientes com IRC tendem a desenvolver, com maior facilidade e devido a progressão das etapas da IRC, a sarcopenia (perda e ou diminuição da força e massa muscular) devido a inatividade física e várias condições patológicas associadas a IRC, tais como: desnutrição, deficiência de vitamina D, acúmulo de toxinas urêmicas, acidose metabólica entre outras.

\section{Classe 2: As limitações e restrições ocasionadas pelo processo hemodialítico.}

A classe 2, intitulada de: As dimensões e restrições ocasionadas pelo processo hemodialítico, representa 12,5\%do total de palavras que foram agrupadas no software IRaMuTeQ. Nesta classe, as palavras de maiores frequência foram, respectivamente: limitação, atividade, imposto, alteração, hemodialítico, realizar, mudança e restrição.

Desta maneira, verifica-se que idoso sem tratamento hemodialítico apresentam algumas limitações de ordem: física, funcional, psicológica, interação social, ansiedade, dentre outras. As limitações de caráter físico geralmente são as que sofrem mais impacto, devido a fragilidade que o idoso adquire durante o próprio processo de envelhecimento (Souza et al., 2017).

De acordo com o dendrograma, foi possível analisar que a classe 2 e 4 são complementares, visto que o tratamento hemodialítico demanda de uma série de restrições associadas à dieta que o idoso precisa manter, e geralmente só há adesão por ser um elemento essencial para manter a vida. Essas restrições podem causar sentimentos de isolamento, tristeza, medo, angústia, relacionadas principalmente a mudança no estilo de vida e grau de dependência, o que podem ser fatores de risco para o desenvolvimento de transtornos emocionais (Ribeiro et al., 2020).

$\mathrm{O}$ tratamento acaba por ocasionar frustações e limitações ao paciente, que devido a condição traz consigo proibições, como manter uma dieta específica que correlacionada a restrições hídricas, delimitando esses pacientes de realizar atividades que antes eram frequentes no seu dia (Ribeiro et al., 2020).

Para Silva et al (2020), o tratamento ocasiona mudanças importantes na rotina do paciente, além de restringir as 
atividades diárias, alimentares e de ingestão hídrica. O tratamento é feito por meio do esquema medicamentoso ininterrupto, além de possuir uma rotina estressante devido a dependência da máquina de hemodiálise. Desta forma, o paciente irá enfrentar mudanças que irão interferir em seu aspecto físico, espiritual, mental e social, necessitando de pessoas que o ajude a enfrentar todas essas barreiras.

\section{Classe 3: Qualidade de vida de pacientes idosos com IRC associado a diálise.}

A classe 3, denominada de qualidade de vida de paciente idosos com IRC associado a diálise, apresenta 25,0\% do total de palavras agrupados no software IRaMuTeQ. Na classe 3 emergiu-se as seguintes frequências de palavras: Qualidade, diálise, padrão, paciente, sintoma, associado, vida e mais. Resulta-se desta forma, que proporcionar a qualidade de vida a pacientes em dialise é de extrema importância para a continuidade do tratamento, pois é através de um instrumento, desenvolvido pela Organização mundial da Saúde (OMS), que se pode proporcionar e mensurar a qualidade de vida de pacientes com IRC em hemodiálise. Outro ponto associado a relevância da qualidade de vida é o avanço das pesquisas no campo em estudo (Costa et al., 2016).

Observou-se também, que a classe 1 e a classe 3 se complementam de acordo com que é ilustrado no dendrograma criado pelos autores, haja vista que os sintomas desenvolvidos em paciente em dialise interfere na qualidade de vida, padrão de vida e nas questões socioeconômicas. Segundo o estudo de Candia et al (2015) sobre a qualidade de vida de idosos em hemodiálise relatou-se sobre uma relação negativa do fator idade, capacidade funcional, aspectos físicos, dor e vitalidade na qual foi avaliado pelo questionário Short Form Health Survey (SF-36), identificando a pior relação da qualidade de vida em idosos do que em jovens adultos.

O questionário SF- 36 é utilizado para avaliar a qualidade de vida de pacientes com IRC na qual possuem itens que avaliam o estado geral da saúde capacidade funcional (CF), aspectos físicos (AF), dor (DOR), vitalidade (VIT), aspectos sociais (AS), aspectos emocionais (AE), e saúde mental (SM). O instrumento demostra um escore de 0 a 100, haja vista que zero corresponde a pior condição de saúde, e 100, a melhor condição de saúde. No entanto, o estudo de Alves et al (2018) mostrou que o escore de aspectos sociais, medido pelo questionário de qualidade de vida, foi o máximo e melhor, indicando que os entraves emocionais e físicos não interfere na vida social de pacientes com IRC.

Em relação a qualidade de vida e o estado nutricional dos idosos com IRC, no estudo de Magalhães et al (2018) é abordado sobre a necessidade de se diagnosticar a desnutrição pois está patologia influencia no risco de infecções, inflamações acentuadas, mortalidade, hospitalizações e diminuição da qualidade de vida de idosos com IRC. Além disso, é imprescindível que os profissionais também deem atenção não somente para os pacientes com destruição, mas também para aqueles com excesso de peso, uma vez que a obesidade é um fator agravante para a lesão renal (Magalhães et al., 2018).

A equipe de enfermagem deve propiciar várias possibilidades para que os idosos com IRC possam identificar fragilidade e as potencialidades relacionadas ao enfrentamento da patologia e do tratamento, tendo em vista o contato direto com o paciente e os familiares. Por isso, o enfermeiro é primordial e responsável por orientar sobre a doença, suas implicações, limitações e os possíveis entraves que possam vim a surgir no decorrer da evolução da IRC (Everling et al., 2016).

\section{Classe 4: A comunicação entre familiares e profissionais para prestação do cuidado.}

A classe 4 nomeada como: A comunicação entre familiares e profissionais para prestação do cuidado, representa $35,9 \%$ as palavras obtidas por meio do software IraMuTeQ. Esta classe apresentou como palavras mais frequentes mostradas no dendrograma: idoso, família, dever, prestar, enfermagem, forma, cuidado e comunicação.

A família e os profissionais da saúde se constituem como grandes influenciadores para o desenvolvimento do autocuidado, por meio da promoção da autonomia e participação do idoso no tratamento, fazendo com que haja melhores 
resultados (Almeida et al., 2019). Contudo, de acordo com Oliveira et al (2020), é a família que constitui a base principal, pois ela possui o papel de ajudar o idoso renal crônico a se adaptar aos novos hábitos e superar suas limitações e restrições, devido o paciente passar a maior parte do tempo com seu familiar.

Uma das maiores dificuldades enfrentadas é a questão da falta da rede de apoio durante o período de transição do tratamento. Essa rede de apoio se constitui principalmente daqueles que são mais próximos ao paciente, como a família e amigos. Assim como o apoio ao doente renal conta bastante para a progressão no tratamento, a falta desse auxílio acaba desestimulando o idoso, fazendo com que ele se sinta triste e rejeitado. Esse sentimento pode levar a sérios problemas psicológicos e expor à situações de perigo por não se importar mais com a continuação da vida (Oliveira et al., 2020)

Segundo Souza et al (2017), pacientes renais crônicos são submetidos a um tempo de tratamento longo, o que reflete na diminuição das atividades diárias e, consequentemente, o idoso acaba ficando dependente devido as limitações físicas que advém da doença. Desta forma, é de grande importância que a rotina da família com o idoso que esteja em tratamento seja conhecida, a fim de que seja traçado um melhor plano de cuidados baseando-se nas particularidades e demandas do idoso e dos familiares (Jacobi et al., 2017).

O enfermeiro consiste no profissional que mais tem contato com o paciente, fornecendo em sua assistência a escuta para melhor acolher o seu paciente. Quando se trata de um paciente com doença crônica, a forma como esse profissional acolhe o indivíduo acaba influenciando na aceitação e adesão do paciente ao tratamento. Desta forma, o profissional consegue se aproximar mais do paciente e sua família, o que reflete em uma melhor elaboração das intervenções que serão realizadas pelo enfermeiro, bem como as orientações ao paciente e cuidadores (Souza et al., 2020).

De acordo com Jacobi et al (2017), o paciente idoso é muito resistente a mudanças, principalmente relacionadas à hábitos e rotinas. Nem sempre a família consegue fazer com que o paciente siga corretamente as restrições feitas devido ao processo de tratamento hemodialítico, o que pode levar a prejuízos ao próprio paciente. Portanto, a comunicação entre família e o profissional enfermeiro é um elemento primordial para que a adesão ao tratamento seja mantida, garantindo sua eficácia.

\section{Conclusão}

Assim, conclui-se que a qualidade de vida de idosos com IRC é um aspecto importante, pois estes pacientes possuem limitações relacionadas a doenças e também ao tratamento por hemodiálise. Por isso, este estudo aborda subsídios, de manejo e identificação, para que profissionais de saúde possam identificar os fatores que interferem na qualidade de vida de idosos com IRC para assim se ter uma evolução positiva e um tratamento adequado.

O evoluir negativo da doença compromete a vida em âmbito global, haja vista que o paciente idoso com IRC, em tratamento hemodialitico, se vê dependente de maquinários, intervenções cirúrgicas, medicamentos, limitações hídricas e alimentares, que não garantem o retorno de sua saúde.

Assim, o estudo ressalva a importância e a necessidade de se conhecer manejo os instrumentos de avaliação da qualidade de vida de idosos com IRC pois é através dessas escalas que é identificados os fatores que interferem nessa qualidade e também no melhor prognostico da doença.

\section{Referências}

Almeida, O. A. E et al. (2019). Envolvimento da pessoa com doença renal crônica em seus cuidados: revisão integrativa. Revista Ciência \& Saúde Coletiva, 24 (5), 1689-1698.

Alves, K. B. et al. (2018). Existe associação entre qualidade de vida e adesão à farmacoterapia em pacientes com doença renal crônica em hemodiálise? Rev. Einstein, $16(1), 1-7$.

Barreto, M. S. et al. (2015). Envelhecimento populacional e doenças crônicas: Reflexões sobre os desafios para o Sistema de Saúde Pública. Rev. Kairós Gerontologia, 18 (1), 325-339. 
Bardin, L. (2016). Análise de conteúdo. 229.

Bowling, C. B, \& Plantinga, L. \& Hall, R. K. et al. (2016). Associação de problemas não específicos de doença com Mortalidade, cuidados de longo prazo e deficiência funcional entre os adultos mais velhos que requerem cuidados de enfermagem especializados após o início da diálise. Clin J Am Soc Nephrol., 11, $2218-2224$.

Camargo, B. V. et al. (2013). IRAMUTEQ: um software gratuito para análise de dados textuais. Temas psicol., 21 (2).

Candia, M. A. B. et al. (2015). Avaliação da qualidade de vida de idosos em hemodiálise pelo questionário KDQOL. Rev. Soc. Bras. Clin. Med.,13 (4), $235-9$.

Costa, G. M. A. et al. (2016). Qualidade de vida de pacientes com insuficiência renal crônica em tratamento hemodialítico. Rev. Enfermaria global, 43 (1), 73.

Clementino, D. C. \& Souza, A. M. Q \& Barros, D. C. C. et al. (2018). Pacientes em hemodiálise: importância do autocuidado com a fístula arteriovenosa. Rev enferm UFPE, 12 (7), 1841-52.

Everling, J. et al. (2016). Eventos associados à hemodiálise e percepções de incômodo com a doença renal. Av Enferm, 34 (1), 48-57.

Ersoy, N. A. \& Akyar, I. (2019). Avaliação multidimensional do prurido em pacientes em hemodiálise. Nephrology. 20 (1), 42.

Fukushima, R. L. M. et al. (2018). Atividade física e a qualidade de vida de pacientes com doença renal crônica em hemodiálise. Fisioter Pesqui, 25 (3), 338 344.

Hirai, K. et al. (2016). Sarcopenia e Inatividade Física em Pacientes com Doença Renal Crônica. Nephrourol Mon.8 (3).

Jesus, N. M. et al. (2019). Qualidade de vida de indivíduos com doença renal crônica em tratamento dialítico. J. Bras. Nefrol. 41 (3).

Júnior, E. V. S. et al. (2018). Impacto dos fatores associados à sintomatologia depressiva na saúde de idosos em hemodiálise. Enfermería Actual de Costa Rica, 35 .

Jacobi, C. S. (2017). A dinâmica familiar frente ao idoso em tratamento pré-dialítico. Revista Escola Anna Nery, 21 (1).

Lira, C. L. O. B. et al. (2015). Coping e qualidade de vida de pacientes em hemodiálise. Estudos Interdisciplinares em Psicologia, Londrina, 6 (1), $82-99$.

Martínez, R. M. \& Gallego, N. \& Cabezas, A. M. et al (2019). Relação entre qualidade de vida relacionada à saúde e ansiedade/depressão em pacientes com hemodiálise crônica. Enferm Nefrol, 22 (3), 274-83.

Marinho, C. L. A. \& Oliveira, J.F. \& Borges, J. E. S et al (2018). Associação entre características sociodemográficas e qualidade de vida de pacientes renais crônicos em hemodiálise. Rev Cuid, 9 (1), 2017-29.

Magalhães, F. G. et al (2018). Impacto de um programa de intervenção nutricional com idosos portadores de doença renal crônica. Rev. Ciência \& Saúde Coletiva, 23 (8), 2555-2564.

Menezes, J. N. R. M. et al (2018). A Visão do Idoso Sobre o Seu Processo de Envelhecimento. Rev. Cont. \& Saúde, 18 (35).

Negreiros, P. I. R. et al (2016). Ações da assistência de enfermagem ao portador de insuficiência renal crônica em tratamento hemodialítico. BIUS, 3 (7).

Organização Mundial da Saúde (2019). OMS divulga metas para 2019; desafios impactam a vida de idosos.

Oliveira, C. R. P. et al (2019). Repercussões da hemodiálise nas atividades básicas e instrumentais de idosos com insuficiência renal crônica. Rev. interscientia, 7 (2), 50-66.

Oliveira, F. A. et al (2020). O processo de transição saúde/doença em pacientes renais crônicos: contribuições para assistência de enfermagem. Revista da Escola de Enfermagem - USP, 54, e03581.

Pretto, C. R. et al (2020). Depressão e pacientes renais crônicos em hemodiálise: fatores associados. Rev. Bras. Enferm, 73 (1), 1-8.

Pereira, R. M. P. et al (2017). Qualidade de vida de idosos com doença renal crônica em tratamento conservador. Rev Bras Enferm, 70 (4), $851-9$.

Parrado, M. C. R. \& Pozo, M. G. \& Garrido, M.C. et al (2017). Análise da qualidade de vida do paciente na fase de pré-dialise. Enferm Nefrol. 20 (3), 233240.

Ribeiro, W. A. \& Jorge, B. O. \& Queiroz, R. S. (2020). Repercussões da hemodiálise no paciente com doença renal crônica: uma revisão da literatura. Revista Pró-UniverSUS, 11 (1), 88-97

Ren, Q. \& Shi, Q. \& Ma, T. et al (2019). Qualidade de vida, sintomas e qualidade de sono de idosos com doença renal em estágio terminal recebendo manejo conservador: uma revisão sistemática. Health and Quality of Life Outcomes, 17 (1), 78.

Richards, C.A. \& Liu, C.F. \& Hebert, P. L. et al (2109). Percepções familiares da qualidade do cuidado de fim de vida para veteranos com CKD avançado. CJASN, $14(1), 1324-1335$.

Santos, K. K. et al (2018). Perfil epidemiológico de pacientes renais crônicos em tratamento. Rev. enferm UFPE online, Recife, 12 (9), 2293 -300.

Souza, P. M. et al (2017). Qualidade de vida de pacientes portadores de insuficiência renal crônica em tratamento de hemodiálise. Rev. Cient. Fac Mais, 6 (4).

Seabra, C. A. M. \& Xavier, S. P. L. \& Sampaio, Y. P. C. C. et al (2019). Educação em saúde como estratégia para promoção da saúde dos idosos: Uma revisão integrativa. Revista Brasileira de Geriatria e Gerontologia, 22 (4). 
Research, Society and Development, v. 10, n. 2, e23610212147, 2021

(CC BY 4.0) | ISSN 2525-3409 | DOI: http://dx.doi.org/10.33448/rsd-v10i2.12147

Sousa, S. S. S. \& Maia, S.F. \& Silveira, F. D. R. et al (2020). Acolhimento do enfermeiro na admissão do paciente renal crônico para tratamento hemodialitico. Rev Fun Care Online, 12, 603-608.

Silva, K. A. L. \& Cargnin, M. C. S. \& Ventura, J. et al (2017). Qualidade de vida de pacientes com insuficiência renal em tratamento hemodialítico. Rev enferm UFPE online, Recife, 11 (11), 4663-70.

Silva, G. L. \& Lemos, K. C. R. \& Barbosa, A. O. et al (2020). Percepção de pacientes renais crônicos em hemodiálise sobre transplante renal. Rev enferm UFPE online, 14, e244498.

Suñer, M. S. \& Soler, R. S. \& Plana, A. M. et al (2020). Qualidade de Vida e Apoio Social das Pessoas em Diálise Peritoneal: Pesquisa de Métodos Mistos. Int. J. Environ. Res. Public Health, 17 (1), 4240.

Tinôco, J. D. S. \& Paiva, M. G. M. N. \& Macedo, B. M. et al (2018). Prurido no paciente em hemodiálise: associação com ingestão de fósforo e nível sérico de cálcio. Rev Gaúcha Enferm, 39.

Ursi, E. S. \& Galvão, C. M. (2005). Prevenção de lesão de pele no perioperatório: revisão integrativa da literatura. Rev. Latino-am. Enferm. Ribeirão Preto, 14 $(1), 124-31$ 